\section{Retention of cardiopulmonary resuscitation skills after hands-only training versus conventional training in novices: a randomized controlled trial}

\author{
Young Joon Kim ${ }^{1}$, Youngsuk Cho ${ }^{1}$, Gyu Chong Cho ${ }^{1 *}$, Hyun Kyung Ji², \\ Song Yi Han' ${ }^{2}$ Jin Hyuck Lee ${ }^{3 *}$ \\ 'Department of Emergency Medicine, Kang Dong Sacred Heart Hospital, Hallym University School of \\ medicine, Seoul, Korea \\ ${ }^{2}$ Department of Emergency Medical Technology, Baekseok University, Cheonan, Korea \\ ${ }^{3}$ Department of Emergency Medicine, Kangwon National University School of Medicine, Chuncheon, Korea
}

Objective Cardiopulmonary resuscitation (CPR) training can improve performance during simulated cardiac arrest; however, retention of skills after training remains uncertain. Recently, hands-only CPR has been shown to be as effective as conventional CPR. The purpose of this study is to compare the retention rate of CPR skills in laypersons after hands-only or conventional CPR training.

Methods Participants were randomly assigned to 1 of 2 CPR training methods: 80 minutes of hands-only CPR training or 180 minutes of conventional CPR training. Each participant's CPR skills were evaluated at the end of training and 3 months thereafter using the Resusci Anne manikin with a skill-reporting software.

Results In total, 252 participants completed training; there were 125 in the hands-only CPR group and 127 in the conventional CPR group. After 3 months, 118 participants were randomly selected to complete a post-training test. The hands-only CPR group showed a significant decrease in average compression rate $(\mathrm{P}=0.015)$, average compression depth $(\mathrm{P}=0.031)$, and proportion of adequate compression depth $(\mathrm{P}=0.011)$. In contrast, there was no difference in the skills of the conventional CPR group after 3 months.

Conclusion Conventional CPR training appears to be more effective for the retention of chest compression skills than hands-only CPR training; however, the retention of artificial ventilation skills after conventional CPR training is poor.

Keywords Cardiopulmonary resuscitation; Retention (psychology); Education; Training
eISSN: 2383-4625

Received: 8 January 2017

Revised: 17 February 2017

Accepted: 28 February 2017

Correspondence to: Gyu Chong Cho Department of Emergency Medicine, Hallym University School of Medicine, Kangdong Sacrid Heart Hospital, 150 Sungan-ro, Seoul, Korea

E-mail:emdrcho@empas.com

Jin Hyuck Lee

Department of Emergency Medicine, Kangwon National University School of Medicine, 1 Gangwondaehak-gil,

Chuncheon 24341, Korea

E-mail: saber99@naver.com

*These two authors contributed equally to this study.

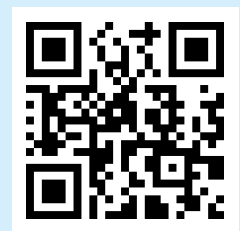

How to cite this article:

Kim YJ, Cho Y, Cho GC, Ji HK, Han SY, Lee JH. Retention of cardiopulmonary resuscitation skills after hands-only training versus conventional training in novices: a randomized controlled trial. Clin Exp Emerg Med 2017;4(2):88-93.

This is an Open Access article distributed under the terms of the Creative Commons Attribution Non-Commercial License (http:// creativecommons.org/licenses/by-nc/4.0/). 


Wapsule
Wummary is already known
Hands-only cardiopulmonary resuscitation (CPR) has been shown to be as effective as conventional CPR and some
studies compared the retention rates of both CPR training methods.
What is new in the current study
Three months after initial CPR training, conventional CPR appears to be more effective in retention of CPR skills than
hands-only CPR.

\section{INTRODUCTION}

Out-of-hospital cardiac arrest (OHCA) is a leading cause of death and a major public health concern. ${ }^{1-3}$ Bystander cardiopulmonary resuscitation (CPR) increases the chances of survival of patients with OHCA. A major meta-analysis reported a mean survival rate of 7.6\% for patients with OHCA after hospital discharge. ${ }^{4,5}$ However, recent epidemiologic data from South Korea indicate that survival rates were only $2.3 \%$ in 2006 to $2007 .{ }^{6}$ To increase bystander CPR rates and improve survival rates of patients with OHCA, nationwide CPR training policies have been established by the Korean Ministry of Health and Welfare and the Korean Association of Cardiopulmonary Resuscitation. Since 2008, practicebased CPR training has been implemented throughout the country. Though this training has been shown to improve skill acquisition, skill retention remains unsatisfactory. ${ }^{8-10}$ More recently, hands-only CPR has been shown to be as effective as conventional CPR, and studies have compared the retention rates of both training methods. ${ }^{8,11}$ To our knowledge, there are no data on skill acquisition or retention after both CPR training methods in South Korean populations. The present study aimed to compare the retention rate of CPR skills in laypersons after hands-only and conventional CPR training.

\section{METHODS}

\section{Study design and subjects}

This study was a randomized controlled trial and was conducted between May and September 2015. Kangdong Sacred Heart Hospital institutional review board approved the study. All participants were volunteers and provided written informed consent. All participants were non-medical college students and they were randomly assigned to either a hands-only or conventional CPR training program. We assessed participants using questionnaires immediately after training and at the 3-month follow-up.

\section{Study protocol}

Participants and instructors were randomly assigned to groups using a computer-generated list. The CPR training had a studentto-instructor ratio of 6:1, with at least 1 manikin for each pair of students. All instructors were doctors, nurses, or emergency medical technicians, who were registered and certified as BLS (basic life support) instructors by the Korean Association of Cardiopulmonary Resuscitation. Training began with an instructional video and was followed by hands-on training with a manikin (Little Anne; Laerdal, Stavanger, Norway). The components of each training program are outlined as follows: (1) hands-only CPR (80 minutes; hands-on practice at recognizing cardiac arrest, seeking help, and performing chest compressions) and (2) conventional CPR (180 minutes; hands-on practice at recognizing cardiac arrest, seeking help, and performing chest compressions and mouth-to-mouth breathing).

At the end of each CPR training program, participants' skills were tested over 2 minutes using the Resusci Anne manikin with a skill-reporting software (Little Anne, Laerdal). In addition, participants were surveyed using questionnaires to assess their confidence and willingness to perform bystander CPR on a 0 to 100 scale.

After 3 months, CPR skills were retested in the same setting without any additional training. Participants were then asked to re-assess their confidence and willingness to perform bystander CPR using the same questionnaire.

\section{Sample size calculation}

We calculated our required sample size using G*Power 3 software (Heinrich Heine University, Düsseldorf, Germany), assuming an alpha of 0.05 and $80 \%$ power. Based on previous studies, which showed a 10\% to 20\% reduction in the proportion of appropriate chest compression depth 6 months post-training, we required 58 subjects per group. ${ }^{8}$ After factoring in a $20 \%$ dropout rate, the total sample size was estimated to be 140 subjects. 


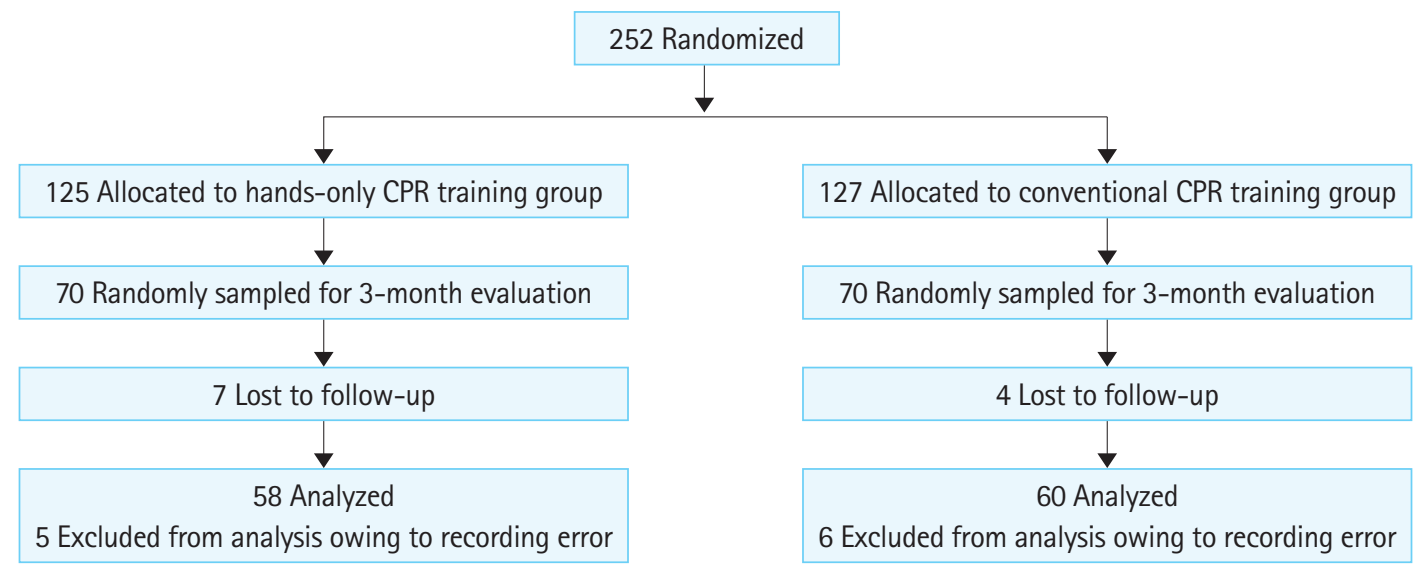

Fig. 1. Flow of study. CPR, cardiopulmonary resuscitation.

Table 1. Characteristics of the 252 participants

\begin{tabular}{lccr}
\hline \multirow{2}{*}{ Characteristics } & \multicolumn{2}{c}{ CPR method } & P-value \\
\cline { 2 - 3 } & Hands-only CPR $(\mathrm{n}=125)$ & Conventional CPR $(\mathrm{n}=127)$ & 0.115 \\
Age & $21.0(19.0-22.0)$ & $20.0(19.0-22.0)$ & 0.456 \\
Sex (male) & $53(42.4 \%)$ & $48(37.8 \%)$ & 0.477 \\
Height $(\mathrm{cm})$ & $168.0(162.0-174.0)$ & $168.0(164.0-175.0)$ & 0.958 \\
Weight $(\mathrm{kg})$ & $57.0(52.0-68.0)$ & $57.0(51.0-68.0)$ & 0.815 \\
Body mass index $\left(\mathrm{kg} / \mathrm{m}^{2}\right)$ & $20.6(19.3-22.5)$ & $20.6(19.3-22.7)$ & 0.135 \\
Prior CPR training (yes) & $38(30.4 \%)$ & $50(39.4 \%)$ & \\
\hline
\end{tabular}

Numbers are medians or interquartile ranges unless stated otherwise.

$\mathrm{CPR}$, cardiopulmonary resuscitation.

Table 2. Comparison of CPR quality in hands-only CPR training group

\begin{tabular}{|c|c|c|c|}
\hline \multirow{2}{*}{ Quality variables during CPR } & \multicolumn{2}{|c|}{ Time of evaluation } & \multirow{2}{*}{ P-value } \\
\hline & Immediately after training $(n=58)$ & 3 Months after training $(n=58)$ & \\
\hline No. of total compressions & $208.0(150.8-227.3)$ & $205.0(180.8-227.0)$ & 0.190 \\
\hline Average compression rate (per min) & $122.0(111.3-130.8)$ & $114.5(101.0-128.3)$ & 0.015 \\
\hline Average compression depth (mm) & $50.5(45.3-56.0)$ & $49.0(42.8-53.0)$ & 0.031 \\
\hline Proportion of adequate compression depth (\%) & $55.6(17.8-98.3)$ & $31.8(5.7-72.4)$ & 0.011 \\
\hline Proportion of inadequate recoil (\%) & $0.0(0.0-0.5)$ & $0.0(0.0-0.4)$ & 0.045 \\
\hline Hands-off time (sec) & $17.5(13.0-34.8)$ & 16.0 (11.0-19.0) & 0.001 \\
\hline
\end{tabular}

Numbers are medians or interquartile ranges unless stated otherwise.

$\mathrm{CPR}$, cardiopulmonary resuscitation.

\section{Statistical analysis}

The data were not normally distributed; thus, nonparametric statistical methods were used. Inter-group comparisons were performed using the chi-square test and the Mann-Whitney U-test for categorical and continuous variables, respectively. The pairwise comparisons of CPR skills immediately after training and at follow-up were conducted using McNemar's test and the Wilcoxon signed-rank test for categorical and continuous variables, respectively. Statistical analyses were performed using IBM SPSS Statistics ver. 23 (IBM Corp., Armonk, NY, USA). A two-tailed value of $\mathrm{P}<0.05$ was considered statistically significant.

\section{RESULTS}

\section{Flow and baseline characteristics}

Of the 252 participants enrolled in the study, 125 were assigned to the hands-only CPR group and 127 were assigned to the conventional CPR group. After follow-up at 3 months, we randomly selected 118 participants to complete a post-training test (Fig. 1). The baseline characteristics of participants are shown in Table 1. There were no significant differences in age, sex, height, weight, body mass index, or prior CPR training between the groups. 
Table 3. Retention of CPR quality in conventional CPR training group

\begin{tabular}{|c|c|c|c|}
\hline \multirow{2}{*}{ Quality variables during CPR } & \multicolumn{2}{|c|}{ Time of evaluation } & \multirow{2}{*}{ P-value } \\
\hline & Immediately after training $(n=60)$ & 3 Months after training $(n=60)$ & \\
\hline No. of total compressions & $141.5(128.0-151.0)$ & $131.0(119.0-142.8)$ & $<0.001$ \\
\hline Average compression rate (per $\mathrm{min}$ ) & $117.5(113.0-124.0)$ & $116.0(108.3-124.8)$ & 0.061 \\
\hline Average compression depth (mm) & $47.5(41.0-52.0)$ & $48.5(41.3-53.0)$ & 0.979 \\
\hline Proportion of adequate compression depth (\%) & $42.9(2.4-86.0)$ & $49.1(6.9-93.0)$ & 0.488 \\
\hline Proportion of inadequate recoil (\%) & $0.0(0.0-0.0)$ & $0.0(0.0-0.0)$ & 0.121 \\
\hline No. of mouth-to-mouth breaths & $8.0(8.0-8.0)$ & $8.0(6.0-8.0)$ & 0.006 \\
\hline Average ventilation volume $(\mathrm{mL})$ & $524.9(165.9-756.0)$ & $297.8(0.0-471.3)$ & 0.025 \\
\hline Proportion of adequate mouth-to-mouth breathing (\%) & $12.5(0.0-50.0)$ & $0.0(0.0-35.4)$ & 0.113 \\
\hline Hands-off time (s) & $49.0(46.0-53.0)$ & $51.0(48.0-57.0)$ & 0.015 \\
\hline
\end{tabular}

Numbers are medians or interquartile ranges unless stated otherwise.

$\mathrm{CPR}$, cardiopulmonary resuscitation.

Table 4. Comparison of CPR quality between the hands-only CPR training and conventional CPR training groups 3 months after training

\begin{tabular}{lccc}
\hline \multirow{2}{*}{ Quality variables during CPR } & \multicolumn{2}{c}{ Methods of training } & P-value \\
\cline { 2 - 3 } & Hands-only CPR $(n=58)$ & Conventional CPR $(n=60)$ & $<0.001$ \\
No. of total compressions & $205.0(180.8-227.0)$ & $131.0(119.0-142.8)$ & 0.728 \\
Average compression rate (per min) & $114.5(101.0-128.3)$ & $48.0(108.3-124.8)$ & 0.767 \\
Average compression depth $(\mathrm{mm})$ & $49.0(42.8-53.0)$ & $49.1(6.9-93.03 .0)$ & 0.301 \\
Proportion of adequate compression depth (\%) & $31.8(5.7-72.4)$ & $0.0(0.0-0.0)$ & 0.104 \\
Proportion of inadequate recoil $(\%)$ & $0.0(0.0-0.4)$ & $8.0(6.0-8.0)$ & - \\
No. of mouth-to-mouth breaths & - & $297.8(0.0-471.3)$ & - \\
Average ventilation volume $(\mathrm{mL})$ & - & $0.0(0.0-35.4)$ & - \\
Proportion of adequate mouth-to-mouth breathing $(\%)$ & - & $51.0(48.0-57.0)$ & $<0.001$ \\
Hands-off time (sec) & $16.0(11.0-19.0)$ & & \\
\hline
\end{tabular}

Numbers are medians or interquartile ranges unless stated otherwise.

$\mathrm{CPR}$, cardiopulmonary resuscitation.

Table 5. Survey on willingness and confidence to perform CPR

\begin{tabular}{|c|c|c|c|c|}
\hline & & \multicolumn{2}{|c|}{ Time of evaluation } & \multirow{2}{*}{ P-value } \\
\hline & & Immediately after training & 3 Months after training & \\
\hline \multirow[t]{2}{*}{ Hands-only CPR training group } & Willingness to perform CPR & $52(98.1)$ & $53(100.0)$ & 1.0 \\
\hline & Confidence score for CPR & $90.0(80.0-100.0)$ & $80.0(70.0-90.0)$ & $<0.001$ \\
\hline \multirow[t]{2}{*}{ Conventional CPR training group } & Willingness to perform CPR & 57 (98.3) & $58(100.0)$ & 1.0 \\
\hline & Confidence score for CPR & $90.0(80.0-100.0)$ & $80.0(69.5-90.0)$ & 0.001 \\
\hline
\end{tabular}

Willingness to perform cardiopulmonary resuscitation (CPR) means number of subjects who have the willingness in each group.

\section{CPR skill variation over 3 months}

Table 2 shows the quality of CPR skills of the hands-only CPR group. At the 3-month follow-up, there was no significant difference in the total number of compressions. However, average compression rate $(P=0.015)$, average compression depth $(P=0.031)$, proportion of adequate compression depth $(P=0.011)$, proportion of inadequate recoil $(P=0.045)$, and hands-off time $(P=0.001)$ had all decreased significantly.

Table 3 shows the quality of CPR skills of the conventional CPR group. There was a decrease in the total number of compressions $(P<0.001)$ at follow-up. There were decreases in the total num- ber of compressions, the number of mouth-to-mouth breaths and the average ventilation volume at follow-up $(P<0.05)$. Conversely, the hands-off time increased at follow-up $(P=0.015)$. None of the other CPR quality parameters had changed significantly after 3 months.

\section{Comparison of CPR skills between hands-only CPR and conventional CPR 3 months after training}

Among the 118 participants selected for CPR skills testing after 3 months, 58 were from the hands-only CPR group and 60 were from the conventional CPR group. The total number of compres- 
sions was significantly greater in the hands-only CPR group (205.0; interquartile range [IQR], 180.8 to 227.0) than in the conventional CPR group (131.0; IQR, 119.0 to 142.8). Average compression rates and depths were similar between the groups (rate: 114.5; IQR, 101.0 to 128.3 vs. 116.0 ; IQR, 108.3 to 124.8 ), (depth: $49.0 \mathrm{~mm}$; IQR, 42.8 to 53.0 vs. $48.5 \mathrm{~mm}$; IQR, 41.3 to 53.0). Similarly, the proportions of adequate compression depth did not differ between the groups (31.8\%; IQR, 5.7 to 72.4 vs. $49.1 \%$; IQR, 6.9 to 93.0$)$. Hands-off time was significantly lower in the handsonly CPR group (16.0 seconds; IQR, 11.0 to 19.0) than in the conventional CPR group (51.0 seconds; IQR, 48.0 to 57.0) (Table 4).

\section{Variation of willingness and confidence in performing CPR} After 3 months, there was no change in willingness to perform bystander CPR in either the hands-only or conventional CPR groups. However, both groups reported a significant reduction in their confidence to perform bystander CPR (Table 5).

\section{DISCUSSION}

The American Heart Association and Korean guidelines for CPR continue to emphasize high-quality CPR. It is recommended that hands-only CPR be used by lay rescuers, whereas conventional CPR is recommended for rescuers who are able to perform rescue breathing. ${ }^{12,13}$ Layperson may be trained in hands-only CPR for adult patients with $\mathrm{OHCA}$ as an alternative to conventional CPR training. ${ }^{14,15}$ Several studies have demonstrated that CPR-specific skills and knowledge deteriorate within 3 to 6 months of initial training. ${ }^{14-16}$ In the present study, we also found that the quality of compression significantly decreased 3 months after hands-only CPR training, but not after conventional CPR training. These results are inconsistent with those of Lim et al.," ${ }^{11}$ who reported skill deterioration in both hands-only and conventional CPR 6 months after initial training. This discrepancy may be explained by differences in follow-up times ( 6 vs. 3 months) and training program duration. In the study by Lim et al., ${ }^{11}$ total training time was 120 minutes, which included 70 minutes of hands-on practice for both the hands-only and conventional CPR groups. However, in the present study, our hands-only CPR training course was only 80 minutes long and included 45 minutes of hands-on practice. On the other hand, our conventional CPR training course was 180 minutes and included 100 minutes of hands-on practice. Several studies have reported that relatively short durations of handsonly CPR training are effective in the acquisition and retention of CPR skills. ${ }^{8,17}$ In contrast, other studies have reported that longer durations of hands-on practice improve the acquisition of CPR skills after training. ${ }^{18,19}$ Our results suggest that, although hands- only CPR may be easier to learn and perform than conventional CPR, it is difficult for lay rescuers to maintain their CPR skills over time. Therefore, a sufficient duration of hands-on practice or proper refresher training may be needed for lay rescuers. In fact, several strategies to promote retention of CPR skills have been suggested, including short and frequent refresher training ${ }_{1}^{20}$ reinforcement using a voice-assisted manikin, ${ }^{21}$ and mobile phonebased reminding. ${ }^{16}$ Further studies are needed to explore effective retention methods for hands-only CPR skills.

Consistent with many previous reports, only $12.5 \%$ of participants in the conventional CPR training group could perform artificial ventilation after approximately 180 minutes of initial training; however, most participants could not properly perform artificial ventilation 3 months after initial training. ${ }^{8,11,16}$ In addition, since the hands-off time for artificial ventilation was significantly increased at follow-up, the number of total compressions significantly decreased in the conventional CPR group. It is known that interrupting chest compression to perform ineffective artificial ventilation is detrimental to the patient's outcome. ${ }^{22}$ Our results suggest that conventional CPR with artificial ventilation performed by laypersons may provide less effective resuscitation in real cardiac arrest situations compared to hands-only CPR. Therefore, it may be reasonable to recommend that lay rescuers perform hands-only CPR for adult victims with OHCA, and perform artificial ventilation only if they are well-trained and have maintained their skills. For the same reason, we believe that handsonly CPR should be advocated as the first-line method of CPR training for lay rescuers.

This study has several limitations that warrant consideration when interpreting our findings. First, the training course time for the conventional CPR group was much longer than that for the hands-only CPR group. The longer training time may affect skill acquisition. However, the purpose of our study was to compare the skill retention rates of both groups. Second, CPR skills were evaluated using a scenario-based simulation test on a manikin. Therefore, our findings may not be applicable to real-life cardiac arrest situations. Third, the participants were young college students, who may have been more capable of acquiring and maintaining CPR skills in comparison to laypersons. Therefore, our findings may not be representative of the general population. Lastly, CPR skills were re-evaluated only once, 3 months after initial CPR training. Further studies should evaluate retention of CPR skills in the longer term.

In summary, conventional CPR training appears to be more effective for retention of chest compression skills compared to handsonly CPR training in the short-term; however, the retention of artificial ventilation skills after conventional CPR training is poor. 


\section{CONFLICT OF INTEREST}

No potential conflict of interest relevant to this article was reported.

\section{ACKNOWLEDGMENTS}

The study was funded by Centers for Disease Control and Prevention of Korea. The funders did not participated in or otherwise influence study.

\section{REFERENCES}

1. Saxon LA. Sudden cardiac death: epidemiology and temporal trends. Rev Cardiovasc Med 2005;6 Suppl 2:S12-20.

2. Mawani M, Kadir MM, Azam I, et al. Epidemiology and outcomes of out-of-hospital cardiac arrest in a developing country-a multicenter cohort study. BMC Emerg Med 2016;16:28.

3. Rea TD, Eisenberg MS, Becker $\sqcup$, Murray JA, Hearne T. Temporal trends in sudden cardiac arrest: a 25-year emergency medical services perspective. Circulation 2003;107:2780-5.

4. Sasson C, Rogers MA, Dahl J, Kellermann AL. Predictors of survival from out-of-hospital cardiac arrest: a systematic review and meta-analysis. Circ Cardiovasc Qual Outcomes 2010;3:63-81.

5. Berdowski J, Berg RA, Tijssen JG, Koster RW. Global incidences of out-of-hospital cardiac arrest and survival rates: systematic review of 67 prospective studies. Resuscitation 2010;81: 1479-87.

6. Ahn KO, Shin SD, Suh GJ, et al. Epidemiology and outcomes from non-traumatic out-of-hospital cardiac arrest in Korea: a nationwide observational study. Resuscitation 2010;81:97481.

7. Lee MJ, Hwang SO, Cha KC, Cho GC, Yang HJ, Rho TH. Influence of nationwide policy on citizens' awareness and willingness to perform bystander cardiopulmonary resuscitation. Resuscitation 2013;84:889-94.

8. Nishiyama $C_{\text {, Iwami }}$, Kitamura $T$, et al. Long-term retention of cardiopulmonary resuscitation skills after shortened chest compression-only training and conventional training: a randomized controlled trial. Acad Emerg Med 2014;21:47-54.

9. Papalexopoulou K, Chalkias A, Dontas I, et al. Education and age affect skill acquisition and retention in lay rescuers after a European Resuscitation Council CPR/AED course. Heart Lung 2014;43:66-71.

10. Avisar L, Shiyovich A, Aharonson-Daniel L, Nesher L. Cardiopulmonary resuscitation skills retention and self-confidence of preclinical medical students. Isr Med Assoc J 2013;15:6227.

11. Lim SH, Aw SJ, Cheong MA, et al. A randomised control trial to compare retention rates of two cardiopulmonary resuscitation instruction methods in the novice. Resuscitation 2016; 103:82-7.

12. Travers $A H$, Perkins GD, Berg RA, et al. Part 3: adult basic life support and automated external defibrillation. 2015 International Consensus on Cardiopulmonary Resuscitation and Emergency Cardiovascular Care Science with Treatment Recommendations. Circulation 2015;132(16 Suppl 1):S51-83.

13. Song KJ, Kim JB, Kim J, et al. Part 2. Adult basic life support: 2015 Korean Guidelines for Cardiopulmonary Resuscitation. Clin Exp Emerg Med 2016;3(Suppl):S10-6.

14. Bhanji F, Donoghue AJ, Wolff MS, et al. Part 14: education. 2015 American Heart Association guidelines update for cardiopulmonary resuscitation and emergency cardiovascular care. Circulation 2015;132(18 Suppl 2):S561-73.

15. Yang HJ, Kim GW, Cho GC, Tak YJ, Chung SP, Hwang SO. Part 8. Cardiopulmonary resuscitation education: 2015 Korean Guidelines for Cardiopulmonary Resuscitation. Clin Exp Emerg Med 2016;3(Suppl):S66-8.

16. Ahn JY, Cho GC, Shon YD, Park SM, Kang KH. Effect of a reminder video using a mobile phone on the retention of CPR and AED skills in lay responders. Resuscitation 2011;82:1543-7.

17. Hirose $T$, Iwami $T$, Ogura $H$, et al. Effectiveness of a simplified cardiopulmonary resuscitation training program for the nonmedical staff of a university hospital. Scand J Trauma Resusc Emerg Med 2014;22:31.

18. Andresen D, Arntz HR, Grafling W, et al. Public access resuscitation program including defibrillator training for laypersons: a randomized trial to evaluate the impact of training course duration. Resuscitation 2008;76:419-24.

19. Lee JH, Cho Y, Kang KH, Cho GC, Song KJ, Lee CH. The effect of the duration of basic life support training on the learners' cardiopulmonary and automated external defibrillator skills. Biomed Res Int 2016;2016:2420568.

20. Nishiyama C, Iwami T, Murakami Y, et al. Effectiveness of simplified 15-min refresher BLS training program: a randomized controlled trial. Resuscitation 2015;90:56-60.

21. Niles D, Sutton RM, Donoghue A, et al. "Rolling Refreshers": a novel approach to maintain CPR psychomotor skill competence. Resuscitation 2009;80:909-12.

22. Kern KB, Hilwig RW, Berg RA, Sanders AB, Ewy GA. Importance of continuous chest compressions during cardiopulmonary resuscitation: improved outcome during a simulated single lay-rescuer scenario. Circulation 2002;105:645-9. 